\title{
Ultrasonic moduli for fluid-saturated rocks: Mavko-Jizba relations rederived and generalized
}

\author{
Boris Gurevich ${ }^{1}$, Dina Makarynska ${ }^{2}$, and Marina Pervukhina ${ }^{3}$
}

\begin{abstract}
Mavko and Jizba propose a quantitative model for squirt dispersion of elastic-wave velocities between seismic and ultrasonic frequencies in granular rocks. Their central results are the expressions for the so-called unrelaxed frame bulk and shear moduli computed under an assumption that the stiff pores are drained (or dry) but the soft pores are filled with fluid. Mavko-Jizba expressions are limited to liquid-saturated rocks but become inaccurate when the fluid-bulk modulus is small (e.g., for gas-saturated rocks). We have derived new expressions for unrelaxed moduli of fluid-saturated porous rocks using Sayers-Kachanov discontinuity formalism. The derived expressions generalize the established Mavko-Jizba relations to gas-saturated rocks, reduce to Mavko-Jizba results when the pore fluid is liquid, and yield dry moduli when fluid-bulk modulus tends to zero. We tested this by comparing our model and the model of Mavko and Jizba against laboratory measurements on a sample of Westerly granite.
\end{abstract}

\section{INTRODUCTION}

Elastic-wave velocities in fluid-saturated rocks at ultrasonic frequencies often are significantly higher than predicted by the Gassmann-Biot theory of poroelasticity. This difference usually is explained by the binary structure of the pore space: relatively stiff pores that occupy the majority of the pore space, and relatively compliant (soft) pores that are present at grain contacts and are responsible for the pressure dependency of the elastic moduli. When a rock is deformed, compliant pores tend to deform much more easily than do stiff ones. In a dry rock, the presence of compliant pores results in substantially lower bulk and shear moduli of the rock compared to a hypothetical rock containing only stiff pores. If the rock is saturated with fluid, the binary structure of the pore space causes significantly different fluid-pressure buildup in soft pores than in stiff pores, and hence large pressure gradients between the two pore types. If the frequency is low enough, the pressure gradient has sufficient time to relax, thus satisfying a crucial assumption of the Gassmann-Biot theory. However, at ultrasonic frequencies, the pressure has no time to equilibrate, effectively making compliant pores isolated from the stiff pores and from each other. This results in higher elastic moduli compared to the low-frequency Gassmann-Biot prediction. This effect is known as squirt dispersion.

Mavko and Jizba (1991) propose a quantitative model for squirt dispersion in granular rocks. An appealing property of their model is that it is based entirely on measurable quantities and does not involve adjustable parameters. The central results of Mavko and Jizba (1991) are the expressions for the so-called unrelaxed frame bulk and shear moduli $K_{u f}$ and $\mu_{u f}$, computed under an assumption that the stiff pores are dry but the soft pores are filled with a fluid. These expressions have the form

$$
\frac{1}{K_{u f}(P)} \approx \frac{1}{K_{h}}+\left(\frac{1}{K_{f}}-\frac{1}{K_{g}}\right) \phi_{c}(P)
$$

and

$$
\frac{1}{\mu_{\mathrm{dry}}(P)}-\frac{1}{\mu_{u f}(P)} \approx \frac{4}{15}\left(\frac{1}{K_{\mathrm{dry}}(P)}-\frac{1}{K_{u f}(P)}\right),
$$

where $K_{\mathrm{dry}}(P)$ and $\mu_{\mathrm{dry}}(P)$ are bulk and shear moduli, respectively, of the dry rock at a given confining pressure $P ; K_{f}$ and $K_{g}$ are bulk moduli of the fluid and of the material of the solid grains, respectively; $\phi_{c}(P)$ is compliant porosity; and $K_{h}$ is the dry bulk modulus of a hypothetical rock without the compliant porosity. Stiff porosity and compliant porosity are assumed to decrease with confining pressure. The stiff-porosity decrease with pressure is assumed to be linear, whereas the compliant porosity is assumed to decrease more quickly at low pressures and to close completely at some pressure $P_{h}$. Thus,

Manuscript received by the Editor 20 October 2008; revised manuscript received 26 January 2009; published online 12 May 2009.

${ }^{1}$ Curtin University of Technology, Department of Exploration Geophysics, Perth, Australia, and CSIRO, Division of Petroleum Resources, ARRC, Perth, Australia.E-mail: B.Gurevich@ curtin.edu.au.

${ }_{3}^{2}$ Curtin University of Technology, Department of Exploration Geophysics, Perth, Australia.E-mail: D.Makarynska@ curtin.edu.au.

${ }^{3}$ CSIRO Division of Petroleum Resources, ARRC,Perth, Australia.E-mail: Marina.Pervukhina@ csiro.au. (c) 2009 Society of Exploration Geophysicists. All rights reserved. 
the linear trend of stiff-porosity decrease can be established from porosity decrease at pressures $P>P_{h}$, and the compliant porosity $\phi_{c}(P)$ at lower pressures can be estimated by subtracting this linear trend from the total porosity $\phi(P)$. The "stiff" modulus $K_{h}$ can be estimated as a dry modulus at pressure $P=P_{h}$. When unrelaxed frame moduli $K_{u f}$ and $\mu_{u f}$ have been computed, they can be substituted into Gassmann's or Biot's equations to obtain saturated (undrained) moduli, as well as compressional and shear velocities.

The predictions of equations 1 and 2 agree well with experimental data (Mavko and Jizba, 1991, 1994; Wulff and Burkhardt, 1997); however these equations have a limitation that stems from the expression on the right side of equation 1 representing the two first terms of the Taylor expansion of $K_{u f}^{-1}$ in powers of $\phi_{c}$. This implies that this approximation is valid only when the second term $\phi_{c}\left(K_{f}^{-1}\right.$ $\left.-K_{g}^{-1}\right)$ is smaller than the first term $K_{h}^{-1}$. Because $K_{g}$ usually is much larger than $K_{f}$, the fluid must be stiff enough that $K_{f} \gg \phi_{c} K_{h}$. This condition is satisfied for typical liquids and most rocks, but might not be valid for gases, and certainly is invalid for dry rocks because $\phi_{c}\left(K_{f}^{-1}-K_{g}^{-1}\right)$ goes to infinity.

In this paper, we provide an alternative derivation of expressions for ultrasonic moduli using discontinuity formalism of Sayers and Kachanov (1995). Our expressions reduce to the equations of Mavko and Jizba when the pore fluid is liquid, but also are valid for gas-saturated rocks. For dry rock, the unrelaxed moduli $K_{u f}$ and $\mu_{u f}$ reduce to the dry moduli $K_{\mathrm{dry}}$ and $\mu_{\mathrm{dry}}$, respectively.

Berryman (2007) uses the general approach of employing SayersKachanov discontinuity formalism to derive Mavko-Jizba equations; Berryman et al. (2002) and Berryman (2006) contain useful insights into the nature of these equations. However, the focus of our study is somewhat different because it is concerned specifically with extending the Mavko-Jizba expressions for unrelaxed isotropic bulk and shear moduli to fluids with arbitrary bulk modulus.

Of course, the transition from the low-frequency (relaxed) to high-frequency (unrelaxed) moduli is gradual and is accompanied by attenuation from wave-induced fluid flow between compliant pores and stiff pores (Mavko and Nur, 1975; O'Connell and Budiansky, 1977; Jones, 1986; Murphy et al., 1986; Dvorkin et al., 1995; Pride et al., 2004). However, like Mavko and Jizba (1991) and Endres and Knight (1997), this paper is concerned exclusively with the unrelaxed moduli, i.e., with the high-frequency limit of the frequency-dependent moduli.

\section{DERIVATION OF THE UNRELAXED FRAME MODULI}

\section{Elastic moduli in terms of displacement discontinuities}

Following Mavko and Jizba (1991) and Shapiro (2003), we assume that the pore space of the rock consists of stiff pores and compliant pores. We further assume that the frequency is so high that the soft pores can be considered to be hydraulically isolated from the stiff pores and from each other. We wish to obtain unrelaxed frame bulk and shear moduli $K_{u f}$ and $\mu_{u f}$, which represent the moduli of the rock whose interconnected stiff pores are dry but whose isolated soft pores can be either dry or filled with a Newtonian fluid. We assume that the dry rock at pressure $P>P_{h}$ contains only stiff pores, and that is an isotropic, linearly elastic solid with a bulk modulus $K_{h}$. At pressure $P<P_{h}$, the rock is weakened by the presence of compliant porosity. We represent the effect of compliant porosity on elastic properties by a system of isotropically distributed displacement dis- continuities at grain boundaries. The effect of such discontinuities on the elastic compliance of a rock $S_{i j k l}$ can be quantified using the formulation of Sayers and Kachanov $(1991,1995)$ :

$$
S_{i j k l}=S_{i j k l}^{0}+\frac{1}{4}\left(\delta_{i k} \alpha_{j l}+\delta_{i l} \alpha_{j k}+\delta_{j k} \alpha_{i l}+\delta_{j l} \alpha_{i k}\right)+\beta_{i j k l} .
$$

Here, $\alpha_{i j}$ and $\beta_{i j k l}$ are second- and fourth-rank tensors that are defined by

$$
\alpha_{i j}=\frac{1}{V} \sum_{r} B_{T}^{(r)} n_{i}^{(r)} n_{j}^{(r)} A^{(r)}
$$

and

$$
\beta_{i j k l}=\frac{1}{V} \sum_{r}\left(B_{N}^{(r)}-B_{T}^{(r)}\right) n_{i}^{(r)} n_{j}^{(r)} n_{k}^{(r)} n_{l}^{(r)} A^{(r)}
$$

where $B_{N}^{(r)}$ and $B_{T}^{(r)}$ are the normal and shear compliance, respectively, of the $r$ th discontinuity in volume $V ; n_{i}^{(r)}$ is the $i$ th component of the normal to the discontinuity; and $A^{(r)}$ is the area of the discontinuity. The symbol $B_{N}^{(r)}$ characterizes the displacement jump normal to the discontinuity produced by a normal traction, whereas $B_{T}^{(r)}$ characterizes the shear displacement jump produced by a shear traction. The discontinuities are assumed to be rotationally symmetric, i.e., $B_{T}^{(r)}$ is assumed to be independent of the direction of the shear traction within the plane of the discontinuity. In equations $3-5$, the cumulative effect of many discontinuities is assumed to be additive. In other words, interaction between discontinuities is neglected (noninteractive approximation, which is valid for a dilute concentration of discontinuities).

Sayers and Han (2002) show that in the case of an isotropic distribution of discontinuities in an isotropic rock, equation 3 gives the following expressions for the isotropic bulk and shear compliances of the rock:

$$
\frac{1}{K_{u f}}=\frac{1}{K_{h}}+3 \alpha+5 \beta
$$

and

$$
\frac{1}{\mu_{u f}}=\frac{1}{\mu_{h}}+2 \alpha+\frac{4}{3} \beta
$$

where $\alpha=\alpha_{11}=\alpha_{22}=\alpha_{33}$ and $\beta=\beta_{1111}=\beta_{2222}=\beta_{3333}$ are diagonal elements of the respective tensors. These tensor elements may be computed for an isotropic distribution of discontinuities by replacing the sums in equations 4 and 5 with integrals over all orientations and taking volume $V$ to be a sphere of radius $R$ :

$$
\alpha=\alpha_{33}=\frac{1}{V} \int_{S_{V}} B_{T} \cos ^{2} \theta d S=\frac{A}{3 V} B_{T}
$$

and

$$
\beta=\beta_{3333}=\frac{1}{V} \int_{S_{V}}\left(B_{N}-B_{T}\right) \cos ^{4} \theta d S=\frac{A}{5 V}\left(B_{N}-B_{T}\right),
$$


where $B_{N}$ and $B_{T}$ are the normal and shear compliance of each plane discontinuity, $S_{V}$ is the surface of all discontinuities in volume $V, A$ is the total area of the surface $S_{V}$, and $\theta$ is angle between the normal to the surface area $d S$ and the $x_{3}$ axis. Substituting these expressions into equations 6 and 7 gives

$$
\frac{1}{K_{u f}}=\frac{1}{K_{h}}+s B_{N}
$$

and

$$
\frac{1}{\mu_{u f}}=\frac{1}{\mu_{h}}+\frac{4}{15} s B_{N}+\frac{2}{5} s B_{T},
$$

where $s=A / V$ is surface to volume ratio of all discontinuities.

\section{Effect of fluid}

Equations 10 and 11 are valid for a rock in which the compliant porosity is either dry or fluid-saturated. For a dry rock, we have

$$
\frac{1}{K_{\text {dry }}}=\frac{1}{K_{h}}+s B_{N}^{\text {dry }}
$$

and

$$
\frac{1}{\mu_{\text {dry }}}=\frac{1}{\mu_{h}}+\frac{4}{15} s B_{N}^{\text {dry }}+\frac{2}{5} s B_{T}^{\text {dry }},
$$

where $B_{N}^{\mathrm{dry}}$ and $B_{T}^{\mathrm{dry}}$ are the dry normal and shear compliances of an individual discontinuity (compliant pore). In turn, unrelaxed moduli of a rock in which compliant pores are fluid-filled can be expressed by equations 10 and 11 , with $B_{N}$ and $B_{T}$ representing saturated fracture compliances. Because in Newtonian fluids the shear stresses are negligible (up to the characteristic frequency of viscous shear relaxation, [Mavko and Nur, 1975]), shear compliance $B_{T}$ is independent of the fluid fill, so that $B_{T}=B_{T}^{\text {dry }}$. Subtracting equation 10 from equation 12 and equation 11 from equation 13 then gives

$$
\frac{1}{K_{\text {dry }}}-\frac{1}{K_{u f}}=s\left(B_{N}^{\text {dry }}-B_{N}\right)
$$

and

$$
\frac{1}{\mu_{\text {dry }}}-\frac{1}{\mu_{u f}}=\frac{4}{15} s\left(B_{N}^{\text {dry }}-B_{N}\right) .
$$

Combining these two equations, we obtain equation 2 , the original Mavko-Jizba equation.

To establish the dependency of $K_{u f}$ and $\mu_{u f}$ on the fluid modulus, we need to know the fluid dependency of normal compliance $B_{N}$, which can be derived in a variety of ways. The simplest, most general way is to use anisotropic Gassmann's equations (Gassmann, 1951; Brown and Korringa, 1975). In general, Gassmann's equations are not valid for an isotropic distribution of isolated compliant pores because pressure in individual pores differs depending on pore orientation. However, because all compliant pores are isolated, $s B_{N}$ can be represented as a sum over all orientations of all soft pores aligned perpendicular to every direction:

$$
s B_{N}=\int_{4 \pi} B_{N} \frac{d s}{d \Omega} d \Omega,
$$

where $d \Omega$ is the solid angle element around a particular direction, and $d s / d \Omega$ is the total area of all soft pores normal to that direction.

In equation $16, Z_{N}=B_{N} d s / d \Omega$ is normal excess compliance caused by all soft pores aligned normal to any particular direction. For a dilute distribution of aligned compliant pores of the same shape, Gassmann's theory is approximately valid because all these pores will have approximately the same pressure induced by uniform deformation, even if they are isolated. Note that dilute concentration already is required by the use of noninteractive approximation of Sayers and Kachanov, equations 3-5. The anisotropic Gassmann's equation for aligned pores gives (Gurevich, 2003)

$$
Z_{N}=\frac{Z_{N}^{\mathrm{dry}}}{1+\frac{K_{f} Z_{N}^{\mathrm{dry}}}{\phi_{c x}\left(1-\frac{K_{f}}{K_{g}}\right)}}=\frac{Z_{N}^{\mathrm{dry}}}{1+\frac{Z_{N}^{\mathrm{dry}}}{\phi_{c x}\left(\frac{1}{K_{f}}-\frac{1}{K_{g}}\right)}},
$$

where $\phi_{c x}=d \phi_{c} / d \Omega$ is the specific volume of compliant pores parallel to the plane normal to $x$ axis (or any other single direction), and $Z_{N}^{\mathrm{dry}}=(d s / d \Omega) B_{N}^{\mathrm{dry}}$. Substituting these expressions into equation 17 gives

$$
Z_{N}=\frac{\frac{d s}{d \Omega} B_{N}^{\mathrm{dry}}}{1+\frac{\frac{d s}{d \Omega} B_{N}^{\mathrm{dry}}}{\frac{d \phi_{c}}{d \Omega}\left(\frac{1}{K_{f}}-\frac{1}{K_{g}}\right)}} .
$$

Noting that

$$
\frac{\frac{d s}{d \Omega}}{\frac{d \phi_{c}}{d \Omega}}=\frac{s}{\phi_{c}},
$$

we obtain

$$
Z_{N}=\frac{\frac{d s}{d \Omega} B_{N}^{\mathrm{dry}}}{1+\frac{s B_{N}^{\mathrm{dry}}}{\phi_{c}\left(\frac{1}{K_{f}}-\frac{1}{K_{g}}\right)}}
$$

and thus

$$
B_{N}=\frac{B_{N}^{\text {dry }}}{1+\frac{s B_{N}^{\text {dry }}}{\phi_{c}\left(\frac{1}{K_{f}}-\frac{1}{K_{g}}\right)} .}
$$

Equation 21 also can be derived from the first-order (dilute) approximation for ellipsoidal cracks based on the Eshelby theorem (Kuster and Toksöz, 1974; Berryman, 1980; Thomsen, 1995). However, the derivation from anisotropic Gassmann's equation (Gurevich, 2003; Berryman, 2007) appears to be slightly more general, in that it does not assume any particular shape of the compliant pores in the plane of the discontinuity. All compliant pores must have ap- 
proximately the same shape, though, or the pressure induced will be different! Also note that equation 21 differs from a similar expression in Hudson's (1981) theory of penny-shaped cracks because it contains the small $1 / K_{g}$ term. Hudson et al. (2001) discusses this minor discrepancy.

Substituting $B_{N}$ as given by equation 21 into equation 10 yields the expression for the unrelaxed drained frame bulk modulus:

$$
\frac{1}{K_{u f}}=\frac{1}{K_{h}}+\frac{s B_{N}^{\mathrm{dry}}}{1+\frac{s B_{N}^{\mathrm{dry}}}{\phi_{c}\left(\frac{1}{K_{f}}-\frac{1}{K_{g}}\right)}} .
$$

The dry compliance can be obtained from the dry modulus using equation 12:

$$
s B_{N}^{\mathrm{dry}}=\frac{1}{K_{\mathrm{dry}}}-\frac{1}{K_{h}},
$$

which gives

$$
\frac{1}{K_{u f}(P)}=\frac{1}{K_{h}}+\frac{1}{\frac{1}{K_{\mathrm{dry}}(P)}-\frac{1}{K_{h}}}+\frac{1}{\left(\frac{1}{K_{f}}-\frac{1}{K_{g}}\right) \phi_{c}(P)},
$$

where as before, $K_{h}=K_{\mathrm{dry}}\left(P_{h}\right)$ is the dry bulk modulus at the highest pressure available.

Equation 24 is the main result of this paper. Note that $\phi_{c}$ usually is very small $(<1 \%$; see Shapiro, 2003); therefore, when the fluid is liquid, $\left(K_{\mathrm{dry}}^{-1}-K_{h}^{-1}\right)^{-1}$ can be neglected compared with $\phi_{c}\left(K_{f}^{-1}\right.$ $\left.-K_{g}^{-1}\right)^{-1}$, and equation 24 reduces to equation 1 , the first of the Mavko-Jizba equations. However, unlike equation 1, equation 24 also is valid when the saturating fluid is a gas. For a dry rock, $\phi_{c}\left(K_{f}^{-1}-K_{g}^{-1}\right)^{-1}$ vanishes and $K_{u f}$ reduces to the dry modulus $K_{\text {dry }}$, as it should. Note that the second of the Mavko-Jizba equations, equation 2, remains unchanged.

As mentioned earlier, Mavko-Jizba equations are particularly useful because they are based entirely on measurable quantities and have no adjustable parameters. The same is true for more general equations 24 and 2 . Indeed, compared with equation 1 , the only new quantity in equation 24 is $K_{\text {dry }}$, which must be known as a function of pressure. However $K_{\mathrm{dry}}$ already is required by equation 2 . Therefore, the combined equations 24 and 2 and the original Mavko-Jizba equations require knowledge of essentially the same information.

The unrelaxed frame moduli $K_{u f}$ and $\mu_{u f}$ are obtained assuming that the stiff pores are dry. The saturated moduli then can be computed using Gassmann's and/or Biot's equations. Specifically, the saturated (undrained) bulk modulus can be obtained by substituting $K_{u f}$ for the frame modulus in Gassmann's equation. The saturated shear modulus will be equal to the unrelaxed frame shear modulus $\mu_{u f}$. If the frequency is equal to or greater than Biot's characteristic frequency $f_{\text {Biot }}$, then the undrained moduli should be substituted into Biot's dispersion equations to obtain compressional and shear velocities as a function of frequency.

Note that Gassmann's and Biot's theories assume that fluid pressure is equalized within the representative volume (RV) of the pore space. This usually is assumed to require that the pore space is inter- connected and that the frequency is low enough to allow sufficient time for pressure relaxation. As previously discussed, higher frequency might prevent equilibration of pressure within one wave period, effectively making the pores hydraulically isolated. However, the condition of interconnected pore space is not a necessary condition for the validity of Gassmann's and Biot's equations. The key condition is the spatially uniform fluid pressure in the pores (within $\mathrm{RV}$ ). In particular, Gassmann's equation is exact for a material with periodically distributed isolated spherical pores because the induced pressure is the same in all these pores (for any frequency below the characteristic frequency of scattering), and it also is quite accurate if the distribution is random. This also is approximately true for all "equant" pores (pores with an aspect ratio on the order $O(1)$ ) (Thomsen, 1985).

Grechka (2008) shows numerically that Gassmann's equation is an excellent approximation for isolated pores of aspect ratio $>0.1$. Therefore, as Mavko and Jizba (1991) suggest, Gassmann's and Biot's equations are applicable to the stiff pores of our system, at seismic and ultrasonic frequencies. This also is consistent with the wellestablished observation that the squirt-flow dispersion between seismic and ultrasonic frequencies is caused mainly by soft porosity and is negligible at high effective stress, where compliant porosity is mostly closed and only stiff pores remain. Indeed, the characteristic frequency of squirt-flow dispersion usually is written as

$$
f_{\text {squirt }}=a^{3} \frac{K}{\eta},
$$

where $a$ is the mean aspect ratio of the pores, $K$ is rock's bulk modulus, and $\eta$ is the fluid viscosity (Jones, 1986). For stiff pores, $a$ $=O(1)$; thus, the squirt frequency is much larger than $1 \mathrm{MHz}$ even for fluids 1000 times more viscous than water.

\section{EXAMPLE}

To illustrate the applicability of our model, we use it to predict high-frequency unrelaxed velocities in a sample of Westerly granite (Coyner, 1984) saturated with three different fluids. Computation of unrelaxed bulk and shear moduli with equations 24 and 2 requires the knowledge of dry bulk and shear moduli $K_{\mathrm{dry}}(P)$ and $\mu_{\mathrm{dry}}(P)$, and of soft porosity $\phi_{c}(P)$ as a function of pressure $P$. In our calculations, the moduli $K_{\mathrm{dry}}(P)$ and $\mu_{\mathrm{dry}}(P)$ are obtained from measurements of dry compressional and shear velocities versus pressure at lab frequency 0.8 to $0.9 \mathrm{MHz}$. The soft porosity $\phi_{c}(P)$ is obtained using a recipe suggested by Mavko and Jizba (1991) as the difference between the measured total porosity $\phi(P)$ and the linear extrapolation of the pressure/porosity trend at high pressures. The Westerly granite sample has porosity of 0.7 to $0.8 \%$, grain density of $2.64 \mathrm{~g} / \mathrm{cm}^{3}$, and a mineral bulk modulus of $56 \mathrm{GPa}$.

To compute the model predictions at each stress, first we calculate the unrelaxed frame bulk $K_{u f}$ and shear $\mu_{u f}$ moduli using equations 24 and 2, assuming that only the soft pores are saturated (the stiff pores are dry). Then, to obtain the saturated compressional and shear velocities, $K_{u f}$ and $\mu_{u f}$ must be substituted into Biot's dispersion equations. However, we note that for such a low-porosity rock ( $\phi$ $\approx 0.8 \%$ ), the Biot and Gassmann predictions will be virtually identical.

Figure 1a and b compares Mavko-Jizba predictions (open symbols) and our model predictions (lines) of P-wave velocity versus pressure for the Westerly granite sample saturated with three different fluids having $K_{f}$ ranging from $0.005 \mathrm{GPa}$ to $2.2 \mathrm{GPa}$. Filled cir- 
cles and diamonds indicate the dry and saturated laboratory measurements, respectively. For the liquid-saturated sample, the predictions of the Mavko-Jizba model (open diamonds) and our model (dashed lines) are identical, and they predict the measured wet velocities (filled diamonds) within 3\% error. However, the two predictions differ substantially for the gas-saturated sample. In particular, for very low fluid modulus $K_{f}=0.005 \mathrm{GPa}$, our model's prediction (solid line) is very close to the dry measurements (solid circles), whereas the Mavko and Jizba (1991) model (open circles) predicts much smaller velocities. This is to be expected because for zero-fluid modulus, the Mavko-Jizba model, equation 1, yields zero unrelaxed bulk modulus, whereas our model, equation 24 , yields the dry modulus.

a)

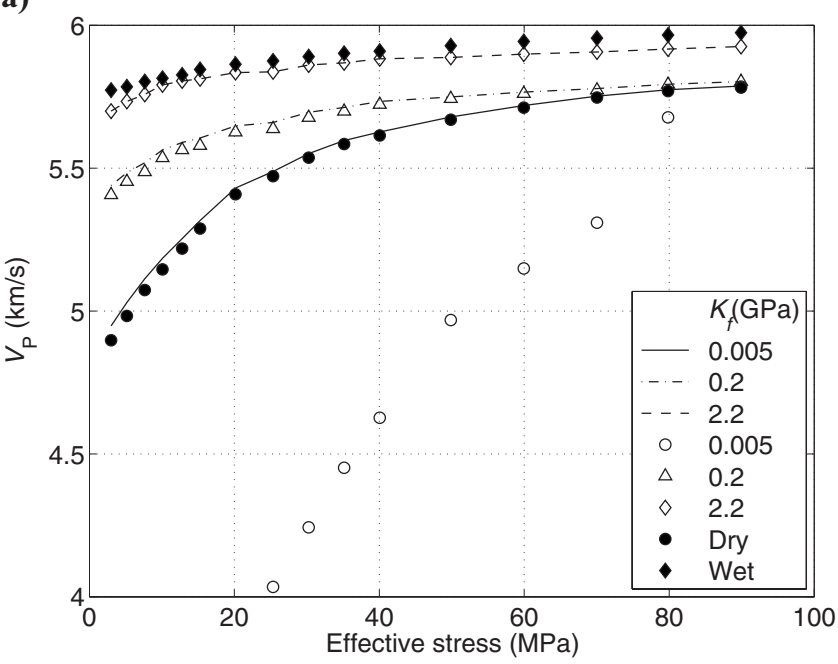

b)

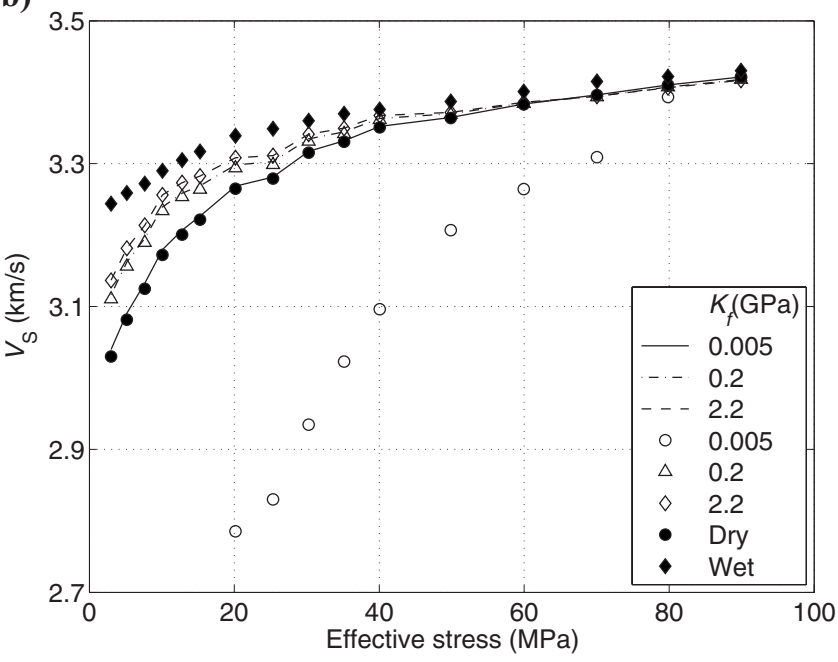

Figure 1. (a) Compressional and (b) shear-wave velocities in a Westerly granite sample as a function of effective stress: laboratory measurements on dry (solid circles) and water-saturated (solid diamonds) sample, predictions of the Mavko and Jizba (1991) model (open symbols), and predictions of the present model (lines) for three different fluids, with bulk moduli shown in the legend.

\section{CONCLUSIONS}

We present a new and concise derivation of expressions for highfrequency bulk and shear moduli of fluid-saturated rocks from pressure dependency of dry moduli and compliant porosity. The derivation is based on the Sayers-Kachanov plane-discontinuity formalism applied to an isotropic distribution of discontinuities. The derived expressions

- generalize the established Mavko-Jizba relations to gas-saturated rocks

- reduce to Mavko-Jizba results when the pore fluid is liquid

- yield dry moduli when the fluid-bulk modulus tends to zero

- contain only measurable quantities

The new expressions are particularly convenient for computations because the same expressions can be used for dry, gas-saturated, and liquid-saturated rocks.

\section{ACKNOWLEDGMENTS}

The authors thank Colin M. Sayers and Serge A. Shapiro for stimulating discussions; Gary Mavko for reviewing the original draft of the manuscript; and the sponsors of Curtin Reservoir Geophysics Consortium (CRGC) for financial support.

\section{REFERENCES}

Berryman, J. G., 1980, Long-wavelength propagation in composite elastic media II. Ellipsoidal inclusions: Journal of the Acoustical Society of America, 68, 1820-1831.

- 2006, Estimates and rigorous bounds on pore-fluid enhanced shear modulus in poroelastic media with hard and soft anisotropy: International Journal of Damage Mechanics, 15, 133-167.

, 2007, Seismic waves in rocks with fluids and fractures: Geophysical Journal International, 171, 954-974.

Berryman, J. G., S. R. Pride, and H. F. Wang, 2002, A differential scheme for elastic properties of rocks with dry or saturated cracks: Geophysical Journal International, 151, 597-611.

Brown, R. J. S., and J. Korringa, 1975, On the dependence of the elastic properties of a porous rock on the compressibility of the pore fluid: Geophysics, 40, 608-616

Coyner, K. B., 1984, Effects of stress, pore pressure, and pore fluids on bulk strain, velocity, and permeability in rocks: $\mathrm{Ph}$. D. thesis, Massachusetts Institute of Technology.

Dvorkin, J., G. Mavko, and A. Nur, 1995, Squirt flow in fully saturated rocks: Geophysics, 60, 97-107.

Endres, A. L., and R. J. Knight, 1997, Incorporating pore geometry and fluid pressure communication into modeling the elastic behavior of porous rocks: Geophysics, 62, 106-117.

Gassmann, F., 1951, Über die Elastizität poröser Medien: Viertel. Naturforsch. Ges. Zrich, 96, 1-23.

Grechka, V., 2008, Fluid-solid substitution in rocks with disconnected porosity: 78th Annual International Meeting, SEG, Expanded Abstracts, 1759-1763.

Gurevich, B., 2003, Elastic properties of saturated porous rocks with aligned fractures: Journal of Applied Geophysics, 54, 203-218.

Hudson, J. A., 1981, Wave speeds and attenuation of elastic waves in material containing cracks.: Geophysical Journal International, 64, 133-150.

Hudson, J. A., T. Pointer, and E. Liu, 2001, Effective-medium theories for fluid-saturated materials with aligned cracks: Geophysical Prospecting, 49, 509-522.

Jones, T. D., 1986, Pore fluids and frequency dependent wave propagation in rocks: Geophysics, 51, 1939-1953.

Küster, G. T., and M. N. Toksöz, 1974, Velocity and attenuation of seismic waves in two-phase media: Part I. Theoretical formulations: Geophysics, 39, 587-606.

Mavko, G., and D. Jizba, 1991, Estimating grain-scale fluid effects on velocity dispersion in rocks: Geophysics, 56, 1940-1949.

- 1994, The relation between seismic $P$ - and $S$-wave velocity dispersion in saturated rocks: Geophysics, 59, 87-92.

Mavko, G., and A. Nur, 1975, Melt squirt in the aesthenosphere: Journal of 
Geophysical Research, 80, 1444-1448.

Murphy, W. F. III., K. W. Winkler, and R. L. Kleinberg, 1986, Acoustic relaxation in sedimentary rocks: Dependence on grain contacts and fluid saturation: Geophysics, 51, 757-766.

O'Connell, R., and B. Budiansky, 1977, Viscoelastic properties of fluid-saturated cracked solids: Journal of Geophysical Research, 82, 5719-5735.

Pride, S. R., J. G. Berryman, and J. M. Harris, 2004, Seismic attenuation due to wave-induced flow: Journal of Geophysical Research, 109, B01201, doi: $10.1029 / 2003 J B 002639$.

Sayers, C. M., and D.-H. Han, 2002, The effect of pore fluid on the stress-dependent elastic wave velocities in sandstones: 72nd Annual International Meeting, SEG, Expanded Abstracts, 1842-1845.

Sayers, C. M., and M. Kachanov, 1991, A simple technique for finding effec- tive elastic constants of cracked solids for arbitrary crac orientation statistics: International Journal of Solids and Structures, 27, 671-680.

-, 1995, Microcrack-induced elastic wave anisotropy of brittle rocks: Journal of Geophysical Research, 100, 4149-4156.

Shapiro, S. A., 2003, Elastic piezosensitivity of porous and fractured rocks: Geophysics, 68, 482-486.

Thomsen, L., 1985, Biot-consistent elastic moduli of porous rocks: Low-frequency limit: Geophysics, 50, 2797-2807.

, 1995, Elastic anisotropy due to aligned cracks in porous rock: Geophysical Prospecting, 43, 805-829.

Wulff, A.-M., and H. Burkhardt, 1997, Mechanisms affecting ultrasonic wave propagation in fluid-containing sandstones under high hydrostatic pressure: Journal of Geophysical Research, 102, 3043-3050. 